\section{El aprendizaje experiencial en la formación docente: un abordaje disruptivo}

\author{
Gloria Mercedes Pacas ${ }^{1}$
}

Palabras clave:

aprendizaje experiencial, formación docente, covid-19, didáctica del inglés, enfoques disruptivos.

\section{Resumen}

Este artículo propone el aprendizaje experiencial como una metodología que puede contribuir sustancialmente a la formación de docentes de idioma inglés en un $\mathrm{El}$ Salvador caracterizado por el autoritarismo, la confrontación, así como el pobre desarrollo de pensamiento crítico y creativo. Luego, se explican algunos de los principios fundamentales de este enfoque, así como las distintas disrupciones que presentan al paradigma de facilitación del idioma inglés en el país. Después, se destacan los aportes que ofrece a la formación de profesionales en la disciplina a través de cuatro experiencias desarrolladas entre 2019 y 2020 con estudiantes y docentes de distintos niveles del Profesorado y la Licenciatura en Idioma Inglés en la Universidad Centroamericana José Simeón Cañas de El Salvador.

\section{Introducción}

Desde el último tercio del siglo $\mathrm{XX}$, la Educación Superior ya reconocía la necesidad de preparar profesionales para el siglo XXI que fuesen competentes en autonomía, ciudadanía y el cuido del medio ambiente (UNESCO-OREALC, 2017). La situación generada por la crisis sanitaria debido al covid-19 ha reafirmado la necesidad de formar seres interculturales, abiertos a perspectivas y saberes diversos que les permitan interpretar y enfrentar la realidad de una manera innovadora e integral. El lenguaje $y$, en concreto, el aprendizaje de un idioma

1 Docente del Departamento de Ciencias de la Educación. Directora de la Licenciatura y el Profesorado en Idioma Inglés de la Universidad Centroamericana José Simeón Cañas. 
global como el inglés juegan un papel fundamental en esta mediación de la realidad.

En dicho contexto, este artículo propone la noción de aprendizaje experiencial (Kolb, 2009) como una metodología que puede contribuir sustancialmente a la formación de docentes de idioma inglés en El Salvador. El texto muestra cómo este enfoque cobra una relevancia importante en el área de formación de docentes de inglés, pues rompe con un abordaje tradicional centrado en el docente, la repetición sin sentido (método audiolingüístico), la estigmatización del error y la memorización de vocabulario junto a reglas gramaticales descontextualizadas (método gramático-traductivo).

En un entorno como el salvadoreño, en el que el discurso dominante llama a la confrontación, la respuesta irreflexiva, el fanatismo y la genuflexión frente al estado de las cosas, el ciclo de aprendizaje constituido por la experiencia concreta, la observación reflexiva, la conceptualización abstracta y la experimentación activa resulta disruptivo y necesario. $\mathrm{El}$ presente texto responde a la pregunta de cómo contribuye el aprendizaje experiencial a una formación de docentes de inglés capaces de abordar y transformar sus contextos educativos de manera creativa, innovadora y crítica. El artículo se construyó a partir de cuatro experiencias desarrolladas entre 2019 y 2020 diseñadas desde el aprendizaje experiencial, las cuales involucraron a estudiantes de distintos niveles del Profesorado y la Licenciatura en Idioma Inglés, así como a docentes de la especialidad en la Universidad Centroamericana José Simeón Cañas.

\section{El aprendizaje experiencial}

El aprendizaje experiencial sucede cuando un estudiante se encuentra personalmente involucrado en una experiencia concreta de aprendizaje vinculada a un interés, necesidad o deseo personal (Haynes, 2007). Es un enfoque en educación que visualiza las expe- riencias de vida de un individuo, sus creencias, educación y trabajo como esenciales en su aprendizaje y comprensión de nuevo conocimiento (Fry et al., 2009; Kolb y Kolb, 2009).

Esta concepción de aprendizaje está basada en el trabajo de varios autores del siglo XX, como John Dewey (18591952), Carl Rogers (1902-1987) y, más recientemente, David Kolb (n. 1939), quienes retoman en sus teorías el aprendizaje a través de la experiencia o el "aprender haciendo" (Bartle, 2015). Dewey plantea la importancia de la resolución de problemas y el análisis crítico en lugar de la memorización y el aprendizaje mecánico. Rogers afirma que el aprendizaje experiencial es significativo y lo contrapone a lo que llamó "aprendizaje cognitivo sin sentido" (Kolb y Kolb, 2009). Kolb estableció que las experiencias concretas de aprendizaje son clave para que este sea significativo. Además, desarrolló el inventario de los estilos de aprendizaje - Learning Styles Inventory (LSI), por sus siglas en inglésorientado a identificar modos de aprendizaje preferidos por las personas (Kolb, 1976).

A pesar de haber sido desarrollado hace ya varias décadas, el aprendizaje experiencial continúa siendo disruptivo. Mientras el modelo dominante de enseñanza del idioma inglés privilegia la experiencia y los intereses del docente, la memorización de datos y la imitación irreflexiva de lo estudiado, el enfoque del aprendizaje experiencial se centra en los intereses del estudiante, la utilización de información para recrear soluciones a problemas comunicativos de la vida real y la reflexión crítica de lo actuado.

Cuando un enfoque de esta naturaleza se emplea en la formación de docentes de inglés, se generan ambientes de aprendizaje significativos, situados - cognitiva y afectivamente estimulantes- que favorecen el desarrollo de habilidades útiles para la vida profesional de los docentes en formación e impacta sus realidades educativas. 


\subsection{Principios fundamentales}

Esta aproximación al aprendizaje tiene cuatro principios fundamentales. El primero de ellos promueve el tránsito de una educación fundamentada en la transmisión de conocimiento —donde el rol del estudiantado es pasivo- a un paradigma donde el estudiante coopera, aprende con y de sus pares mientras se encuentra involucrado en experiencias ancladas en el mundo real (Kolb, 1984). Este principio es el del diseño de experiencias centradas en el estudiante y no en el docente.

El segundo principio contempla una nueva perspectiva del rol del docente. En el aprendizaje experiencial, la función del docente consiste fundamentalmente en diseñar la experiencia y facilitar el proceso, el cual debe ser flexible y semiestructurado (Kolb, 1984). Se procura la interacción entre pares, a modo de que las distintas dinámicas permitan aprender del y con el otro. Así, se desarrolla la flexibilidad cognitiva, definida como "la capacidad de cambiar una estrategia o respuesta por otra para enfrentar de forma más eficiente y flexible un problema o situación específica, lo que implica un permanente monitoreo o evaluación y ajuste de acuerdo con los resultados y opciones disponibles" (Rojas-Barahona, citado por Badillo Torres, 2021).

Un tercer principio es que las experiencias diseñadas deben desarrollar habilidades socioemocionales. Estas se conciben como "aquellos conocimientos y destrezas que nos ayudan a conocer y manejar de mejor forma nuestras emociones, formar nuestra identidad, colaborar con los demás, tener relaciones positivas con las personas, tomar decisiones responsables, manejar situaciones retadoras y respetar las normas" (MINEDUCYT, 2020).

El cuarto pilar fundamental es la reflexión y el análisis crítico sobre lo actuado y lo aprendido. Esto se convierte en un continuo que permite alcanzar nuevos aprendizajes, desarrollando capacidades reflexivas y de análisis (Ambrose et al., 2010; Cooper et al., 2010). Además, se destaca que, durante las experiencias, el estudiante está activamente involucrado en todo el proceso: cuestionando, experimentando, resolviendo problemas, tomando iniciativa, responsabilidad, expresando su creatividad y construyendo significados (Bartle, 2015). Asimismo, se fomenta que el aprendiente se relacione consigo mismo, con los demás y con el mundo exterior. En ese sentido, el diseño mismo de la experiencia contempla la posibilidad de aprender de los errores, los aciertos, la incertidumbre y la ambigüedad en un contexto seguro y apoyado por el docente, por sus pares y sus propias reflexiones.

\subsection{El ciclo del aprendizaje según Kolb}

El aprendizaje experiencial involucra una serie de fases (ver figura 1). Kolb (1984) desarrolló un ciclo de cuatro etapas que plantea un continuo de secuenciación didáctica de la experiencia de aprendizaje orientado a profundizar distintos tipos de habilidades. La primera etapa es la experiencia concreta, mediante la cual se desarrolla la capacidad de aprender experimentando. La segunda etapa es la observación reflexiva, que expone al estudiante a aprender reflexionando desde la experiencia misma, vinculando lo realizado a los resultados obtenidos. La tercera es la conceptualización abstracta, durante la cual el estudiante, a partir de la reflexión, genera conclusiones más amplias y aplicables a otros contextos. La última es la experimentación activa, en la cual el estudiante aplica sus teorías y nuevos aprendizajes para resolver problemas de la realidad vinculados a la experiencia observada y reflexionada. En este proceso, la teoría y la práctica se conceptualizan y reconceptualizan, en una espiral de mayor profundidad y comprensión (Kolb y Fry, 1975). 
Figura 1. El ciclo de Kolb

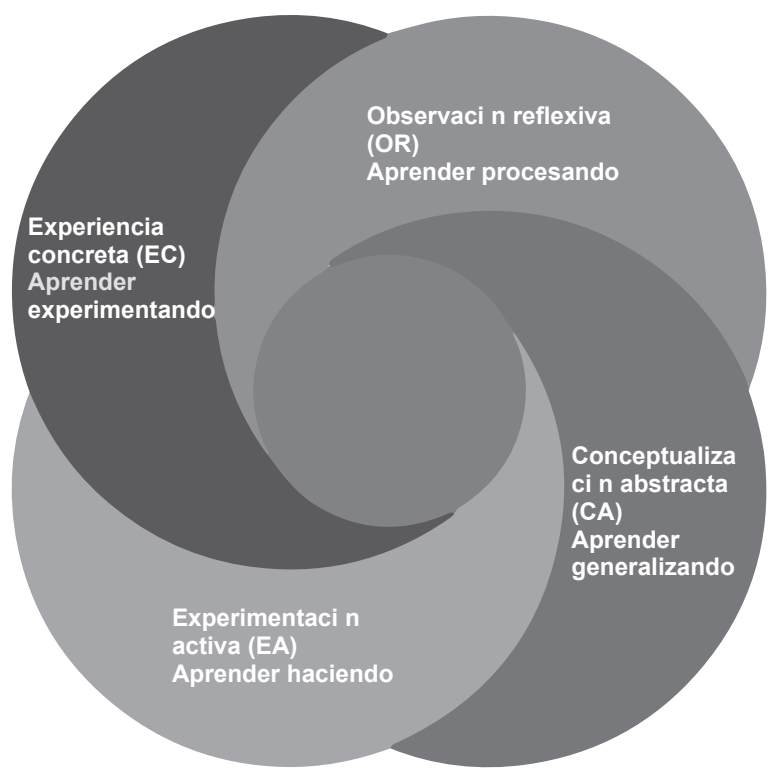

Fuente: elaboración propia con base en Kolb (1986).

Tal y como lo plantean Cooper et al. (2010), el aprendizaje inicia con experiencias que les permiten a los participantes observar, revisar y reflexionar sobre lo que han practicado para luego analizar de manera crítica, vinculando así sus experiencias a una teoría o a vivencias previas. Es así como una experiencia de aprendizaje involucrando las cuatro fases del ciclo implica la exposición a procesos que promueven la flexibilidad cognitiva, la reflexión, el análisis crítico y la atención multisensorial a los estilos de aprendizaje.

\section{Aportes del aprendizaje experiencial a la formación de docentes de inglés}

La realidad en los centros educativos de El Salvador actual no es muy distinta a la de hace décadas. Año con año, educadores de inglés en formación y sus docentes nos encon- tramos con el peso de un paradigma que tiene al profesorado como centro de su quehacer: nada sucede, nada se detiene si no es bajo su dirección. Aunque haya sido formado con un enfoque comunicativo -el cual presupone el uso del idioma meta para resolver necesidades de comunicación- la utilización del inglés está ausente en sus clases. Así, recurre a técnicas como la repetición en coro o la anotación de listas de vocabulario con su respectiva traducción al español y considera que lo que aprendió en la universidad no puede ser aplicado a su contexto educativo.

Las justificaciones para mantenerse en el modelo dominante van desde el alto número de estudiantes y la falta de interés en su materia, hasta el poco acceso a recursos didácticos y tecnológicos, el bajo involucramiento de los padres y las madres de familia y el casi nulo apoyo por parte de las autoridades escolares. Así, a lo largo del ejercicio 
docente, se han identificado cuatro tendencias en el modelo de docencia dominante: el diseño de secuencias didácticas centradas en el docente, las clases expositivas, el uso excesivo del libro de texto y la escasa reflexión docente sobre la propia práctica pedagógica. Las experiencias desarrolladas entre 2019 y 2020 permitieron constatar los aportes del aprendizaje experiencial a la formación de docentes de inglés, como se detalla a continuación.

\subsection{De las secuencias didácticas centradas en el docente al diseño de secuencias didácticas centradas en el estudiante}

La primera tendencia dominante encontrada en las clases de inglés de varios centros educativos del país es la secuenciación didáctica centrada en el docente. Esto significa que es este quien define lo que debe aprenderse, cómo y mediante qué recursos, ya que es la figura central del proceso educativo. El estudiante es un receptor pasivo de las propuestas pedagógicas del docente y tiene poco o nulo control sobre cómo se facilita su aprendizaje. En el caso de los bachilleratos técnicos, este paradigma es aún más pronunciado. La necesidad de aprender "inglés técnico" es usualmente abordada por el docente mediante la reproducción de glosarios según la especialidad (Turismo, Electrónica, Contabilidad o Salud). La clase se limita a la memorización de conceptos considerados relevantes y su correspondiente traducción en español. Además, el aprendizaje del idioma es abordado desde una visión utilitarista, pues al futuro trabajador se le prepara para responder a las demandas del contexto laboral, que pueden ser tan reducidas como comprender un manual para hacer funcionar un circuito eléctrico o memorizar frases para atender a un turista extranjero de habla inglesa.

En contraste, el aprendizaje experiencial desarrolla en los futuros docentes de inglés una flexibilidad cognitiva y capacidad reflexiva que los lleva a experimentar con el diseño de secuencias didácticas y recursos de aprendi- zaje centrados en el estudiante. Estos atienden sus diversas formas de aprender, planteándose el desarrollo de la dimensión cognitiva, socioemocional y multisensorial de sus estudiantes. Además, requiere que estos cooperen y aprendan entre sí a través de experiencias de aprendizaje asociadas a necesidades comunicativas del mundo real.

El proyecto Centro Óscar Arnulfo Romero-UCA (COAR), realizado entre los meses de agosto-octubre de 2019, logró evidenciar que cuando el aprendizaje se basa en el estudiante, es posible fomentar el uso comunicativo del idioma, implementar estrategias para atender a grupos numerosos, utilizar efectivamente los recursos accesibles y aumentar la motivación hacia el aprendizaje del inglés.

Este proyecto requirió un alto nivel de coordinación y trabajo colaborativo tanto entre docentes del equipo como entre el estudiantado del Profesorado en los tres niveles de formación (primero, segundo y tercer año). Se diseñó el proceso, el cual requirió la obtención de los programas de Inglés Técnico del COAR. Luego, en colaboración con el equipo docente de la especialidad, se formaron once grupos de tres estudiantes para atender necesidades específicas de los Bachilleratos Técnicos en Electrónica, Contabilidad, Salud y Turismo. Se establecieron tres roles en cada grupo: a) el estudiante de segundo año fue el docente responsable del diseño de la secuencia didáctica a implementar, así como de los recursos a utilizar. Estaba cursando Didáctica del Inglés I, cuyo enfoque eje es el aprendizaje experiencial; b) el estudiante de tercer año era el asesor y había vivenciado el aprendizaje experiencial el año anterior. Se involucró activamente apoyando al estudiante de segundo año retroalimentando y enriqueciendo el plan presentado; c) el estudiante de primer año ayudó en el diseño de recursos didácticos, aportó ideas, aprendió de sus pares. Dado que aún no había cursado ninguna de las didácticas de la especialidad, la experiencia se convirtió en un proceso que le permitió experimentar con los marcos 
teóricos y las metodologías que estudiaría el año siguiente.

Luego de finalizar la planificación, en la universidad se simuló la secuencia didáctica y el uso de recursos didácticos diseñados durante jornadas en que participaron los tres años de formación. En estas, se retroalimentó lo presentado y se hicieron los ajustes necesarios. Finalmente, los tres años del Profesorado en Inglés, junto con sus docentes, visitaron el COAR y facilitaron once talleres de inglés técnico a grupos de tres a cinco estudiantes. Cada taller tenía las siguientes características: a) lenguaje técnico metacontextualizado en situaciones del mundo real (¿qué debía poder comunicar el estudiante que no sabía hacer al inicio de la sesión?); b) recursos didácticos diseñados para los distintos estilos de aprendizaje; c) momentos de trabajo individual y de trabajo colaborativo entre pares. Durante la jornada, se pudo constatar el alto nivel de motivación por parte de los estudiantes atendidos, quienes afirmaban haber aprendido de una forma que les había resultado divertida y les había demostrado la posibilidad de usar el idioma inglés con fines comunicativos.

La experiencia en el centro educativo visitado evidenció en el estudiantado del COAR una alta motivación y habilidad de comunicación al finalizar los talleres de inglés técnico. Esto favoreció el desarrollo de sus competencias lingüísticas, rompiendo con la perspectiva de que ser docente de inglés es seguir un libro de texto y hacer que se llene un libro de trabajo. Desde la perspectiva de formadora de futuros formadores, la actividad demostró que el aprendizaje experiencial tiene mucho que aportar a la didáctica del inglés, sobre todo, a estas áreas del llamado inglés técnico, las cuales siguen siendo facilitadas desde un enfoque memorístico e irreflexivo. ${ }^{2}$

\subsection{De la clase expositiva a la atención de distintos estilos de aprendizaje}

Al visitar distintos centros educativos, nos encontramos con una metodología que privilegia el uso de la clase expositiva en muchas asignaturas. En particular, la clase de inglés parece haber quedado fosilizada en la época de los años sesenta y setenta, una era en que método gramatical-traductivo y el método audiolingüístico prevalecían en las clases de Inglés en todo el mundo (Harmer, 2001). El primero se vivencia cuando encontramos a docentes presentando enunciados en idioma inglés para ser traducidos al español, explicando reglas gramaticales y pidiendo a los estudiantes que memoricen listas de vocabulario en inglés y su significado en español. El segundo se concretiza en el aula cuando el docente produce oralmente un enunciado en idioma inglés y pide a los estudiantes que repliquen el modelo (tanto en pronunciación como en entonación).

En su tiempo, audiolingualismo fue un método disruptivo, pues en lugar de hacer que el estudiante aprendiera sobre una estructura gramatical y tradujera desde el idioma natal, este implicó que al menos el docente utilizara el idioma meta como modelo a repetir por los y las estudiantes. El objetivo de este método era la eliminación del error en la imitación del patrón modelado por el docente. Las frases se repetían una y otra vez, hasta conseguir perfección en pronunciación, entonación, cadencia y acento. Todas estas prácticas permanecen en la clase de inglés y han generado muchos problemas al uso comunicativo

2 Paralelamente, se realizó una campaña de recolección de libros y recursos didácticos para donar al COAR. Se recolectaron 153 títulos, 78 CD y CD-Rom y se entregaron las planificaciones y recursos didácticos del idioma inglés diseñados para los Bachilleratos en Salud, Turismo, Electrónica y Contabilidad. Esto fue relevante de cara al aprendizaje centrado en el estudiante, puesto que se realizó a iniciativa de personas en distintos niveles de formación. Evidenció capacidad de agencia y liderazgo para lograr una donación de recursos pensados en el grupo meta. 
del idioma. Por una parte, el estudiante ha introyectado que el error (lejos de ser parte del proceso de aprendizaje de cualquier habilidad) será penalizado, por lo que su ansiedad y rechazo al idioma inglés, a su vez, va en detrimento de su motivación. Por otra, el docente (consciente o inconscientemente) se concibe como el modelo a seguir y privilegia la clase expositiva, con largas explicaciones de las reglas gramaticales y recitaciones de enunciados descontextualizados como única estrategia para controlar al grupo y hacer que, al menos, este se enfoque en la lección del día.

Lo anterior se da a pesar de que diversos estudios señalan que en un grupo predominará el estilo kinestésico (aprende mejor si hay movimiento involucrado) y visual (se involucra más si hay imágenes, diagramas, mapas conceptuales ilustrando un concepto). Planificar clases con un predominio de lo expositivo es tomar en cuenta a una minoría de aprendices auditivos (aprenden mejor mediante la escucha), la cual se estima no mayor al 3 \% (Harmer, 2001). Esto sería el equivalente a preparar un gran banquete para invitados carnívoros, cuando el $97 \%$ es vegetariano.

En contraste, docentes de inglés que han sido formados en aprendizaje experiencial logran, a través de la atención a distintos estilos de aprendizaje (visual, auditivo, kinestésico y táctil), la reducción del filtro afectivo (manteniendo niveles de ansiedad bajos), lo cual aumenta la motivación y el aprendizaje del idioma con una perspectiva comunicativa: el idioma se aprende para expresar ideas $y$ sentimientos que nos son significativos; el error es parte importante del proceso de aprendizaje (Krashen, citado por Carston, 2020). Por otro lado, aun cuando la teoría de los estilos de aprendizaje está siendo cuestionada (Reiner y Willingham, 2010), es innegable que entre más variadas sean las estrategias de enseñanza implementadas, mejores posibilidades habrá de que el aprendizaje sea significativo y motivador. En las asignaturas de Didáctica del Idioma Inglés I y II, se hace especial énfasis en un diseño de secuencias didácticas que involucren técnicas, recursos y estrategias que estudiantes con distintos estilos de aprendizaje encuentren motivantes. Esto se ha logrado aun en un contexto en el que las prácticas docentes han migrado a la modalidad virtual debido a la crisis sanitaria mundial. En el siguiente vínculo, se ofrece un ejemplo de la planificación de una clase realizada por una estudiante de tercer año del Profesorado en Inglés en abril de 2020: https://docs.google.com/document/d/1DU01z3Rc4ujtyVjIq5lkHwzx5plibilp/edit?usp = sharing\&ouid = $108946069922585521675 \&$ rtpo$\mathrm{f}=$ true\&sd=true. En ella se observan también cómo los recursos diseñados desde el aprendizaje experiencial aportan a la formación de un docente que tome en cuenta la diversidad de su estudiantado.

La secuencia didáctica planificada responde a lo propuesto por el aprendizaje experiencial en varios sentidos. Por una parte, el objetivo está claramente planteado desde la perspectiva del estudiante: lo que este será capaz de hacer con el lenguaje-meta, que en este caso son sustantivos contables y no contables. Además, el objetivo está vinculado al mundo real: se espera que el estudiantado pueda crear una conversación ya sea en un restaurante, en el supermercado o en el hogar, usando el lenguaje estudiado. Por otro lado, la columna interaction patterns (patrones de interacción) visibiliza la centralización en el estudiante (¿quién está haciendo qué?): T-Ss significa que es el docente quien se está dirigiendo al grupo; Ss-T, los estudiantes al docente; Ss-Ss, los estudiantes están interactuando entre sí, utilizando el lenguaje-meta con propósitos comunicativos. Esto posibilita hacer ajustes pertinentes al momento de planificar, puesto que si, por ejemplo, el patrón de interacción resulta ser en su mayoría T-Ss, sería un indicador de que el docente está abusando de la clase expositiva; por el contrario, Ss-Ss significa que hay mucha interacción entre pares y se está logrando que haya mucho más uso del idioma por parte de estos. 
En cuanto a la atención de los distintos estilos de aprendizaje, la columna VAKT significa visual, auditory, kinesthetic and tactile. Así, se visibiliza qué tanto el diseño de materiales $y$ actividades han considerado a estudiantes con distintos estilos. En el ejemplo anterior, la docente en formación hizo su práctica docente en modalidad virtual, por lo que diseñó actividades interactivas con la herramienta digital Genially, cuyo uso fue abordado en la clase de Didáctica del Inglés. Aun cuando es la misma herramienta, la utilizó de maneras variadas y de forma muy creativa. Además, usó videos y pizarra digital. Cada recurso está dirigido a que estudiantes visuales, auditivos, kinestésicos y táctiles sean estimulados en distintos momentos de la secuencia. Tenemos, pues (refiriéndonos a la metáfora anterior), un menú con comida vegana, vegetariana y carnívora para cada tipo de invitado.

Como docente, las experiencias relacionadas con el diseño de secuencias didácticas y recursos dirigidos a diferentes estilos de aprendizaje han develado la inmensa capacidad de agencia y creatividad de los futuros docentes de inglés. En una época de tanta incertidumbre, la cual ha puesto en riesgo la vida misma del estudiantado atendido, este ha demostrado que un enfoque de formación desde el aprendizaje experiencial desarrolla la habilidad de adaptarse a contextos emergentes y permite seguir facilitando el idioma en entornos virtuales, mientras dan uso pedagógico óptimo a distintas herramientas digitales para que sus estudiantes alcancen los objetivos de aprendizaje y continúen su formación.

\subsection{De usuarios de un libro de texto a creadores de sus procesos}

El rol del docente de inglés como simple usuario de un libro de texto y libro de trabajo de alguna editorial con la cual la institución tiene un acuerdo comercial es otra tendencia que domina los centros educativos. Las razones van desde un acomodamiento por parte del mismo docente, la presión de la administración escolar porque se utilice, hasta la consecuente exigencia por parte de los padres y las madres de familia para que se le saque provecho a su inversión. Tal como sucede en otras asignaturas, bien utilizados, los libros de texto pueden resultar un apoyo invaluable tanto para docentes como para estudiantes. Sin embargo, lo que encontramos en algunos centros educativos es una aplicación acrítica de un currículo que no necesariamente está diseñado para un contexto como el nuestro. Por ejemplo, hay textos que han sido pensados para jóvenes que aprenden inglés en un país de habla inglesa, otros para adultos migrantes, algunos con enfoques metodológicos desfasados o respondiendo a otras realidades.

Los futuros docentes de inglés que están siendo formados con un enfoque de aprendizaje experiencial y que participan de estas experiencias se conciben como creadores de sus procesos didácticos. Asumen que pueden incorporar actividades y recursos planteados por el libro de texto, pero el proceso no gira en torno a este. De esa manera, favorece el desarrollo de competencias lingüísticas de sus estudiantes, rompiendo con la perspectiva de que ser docente de inglés es seguir un libro de texto y hacer que se llene un libro de trabajo.

Como se señaló anteriormente, el aprendizaje experiencial cobra especial importancia en tiempos de pandemia por el covid-19. Durante el año 2021, estudiantes de la asignatura Modalidad Virtual para el Aprendizaje del Idioma Inglés diseñaron aulas virtuales y recursos para el desarrollo de habilidades receptivas (comprensión lectora y comprensión auditiva) y habilidades productivas (expresión oral y expresión escrita). El grupo en mención es graduado del Profesorado en Idioma Inglés para Tercer Ciclo de Educación Básica y Educación Media por la Universidad Centroamericana José Simeón Cañas y se encuentra en su último año de la Licenciatura en Idioma Inglés. Comparten entonces una formación inicial docente basada en el aprendizaje experiencial, además de experiencia docente en la mayoría de los casos. 
El proyecto final en la mencionada asignatura consistió en que cada estudiante debía diseñar el syllabus de un curso en línea con sus respectivas aulas y recursos virtuales de aprendizaje para las cuatro macrohabilidades lingüísticas. Aun cuando podían tomar de referencia el libro de texto utilizado en sus instituciones educativas, se les pidió que construyeran su proceso incorporando las herramientas digitales aprendidas a lo largo del ciclo académico. Las propuestas resultantes del ejercicio comparten varias características: a) son diseñadas en base al aprendizaje experiencial, b) tienen el enfoque comunicativo al centro (el idioma se aprende para expresar y comprender ideas que les son significativas, c) hay uso pedagógico de las TIC, d) usan el learning design (también conocido como diseño instruccional) para el diseño del syllabus del curso. El diseño instruccional está definido como la planificación deliberada e intencional de experiencias de aprendizaje que potencien el desarrollo de habilidades en el estudiantado y la creación de experiencias motivadoras (Pilbeam et al., 2012). Algunos componentes esenciales de este enfoque de diseño son el análisis de la audiencia (¿quiénes son mis estudiantes?), la armonización de los objetivos de aprendizaje con los recursos a diseñar y los instrumentos de evaluación, así como el uso de recursos multimedia y herramientas digitales.

En el siguiente vínculo se puede visualizar un ejemplo de syllabus presentado por una de las estudiantes: https://docs. google.com/document/d/1gV95ZpG_XJfWUd3AG0T5777RmZ7aShghqxEOEX8pOA/ edit?usp=sharing. Se puede constatar cómo se ha logrado el uso óptimo del libro de texto como base para el diseño de sus propios procesos. Por ejemplo, aunque la unidad se titula I used to go to New York on Vacation ("Solía ir a Nueva York para vacaciones"), la estudiante sitúa la experiencia en la realidad de sus estudiantes al proponerles la creación de un glogster (herramienta que permite crear infografías multimedia) narrando sus propias vivencias. Además, aborda las experiencias de aprendizaje desde un enfoque comunicativo, al solicitarles el uso de estructuras gramaticales para narrar experiencias que les son importantes. Por otro lado, su aula virtual expone a sus estudiantes a una variedad impresionante de recursos virtuales de aprendizaje diseñados por ella misma específicamente para su grupo.

La experiencia en esta asignatura da cuenta de los aportes del aprendizaje experiencial para deconstruir paradigmas que ponen al centro del proceso el uso de un libro de texto. En su lugar, este enfoque promueve la construcción de procesos de formación de profesionales en idioma inglés, quienes inician con un análisis de su realidad, del perfil de sus estudiantes y de los objetivos de aprendizaje que se aspira alcanzar. Luego, se transita al diseño de syllabus y recursos didácticos alineados con las conclusiones de dicho análisis. Para que todo esto se logre, el desarrollo de pensamiento crítico y creativo, así como el concebirse agentes de transformación de su realidad educativa, se vuelve fundamental.

Es importante destacar que este no es un proceso libre de dificultades, sobre todo cuando se tiene que lidiar con exigencias institucionales, se afectan intereses económicos de las editoriales y el mismo docente de inglés ha introyectado un rol bastante pasivo frente a dicho contexto. Ha sido, por tanto, aún más satisfactorio constatar que desde el aprendizaje experiencial se puede lograr una reconceptualización del uso adecuado del material de apoyo y redescubrir la capacidad de innovación y creatividad de nuestro estudiantado.

\subsection{De la práctica irreflexiva a la práctica reflexiva según el ciclo de Kolb}

Una constante en la práctica del docente de inglés en nuestros centros educativos es la sobrecarga de responsabilidades y grupos a atender, así como horarios dispersos a lo largo de la jornada laboral. Esto es extensivo a docentes de otras disciplinas; sin embargo, en el caso del docente de lengua extranjera, se vuelve especialmente difícil, considerando 
que, además, en cada grupo de estudiantes suele haber una diversidad de niveles de dominio del idioma. Esta problemática genera una dinámica intensa en la que apenas queda espacio para reflexionar sobre la práctica, analizar el abordaje y replantearse lo actuado.

Por el contrario, desde el aprendizaje experiencial, se generan entornos educativos que promueven la práctica reflexiva, el análisis y el cuestionamiento de sus valores en relación con la docencia. Dewey entiende la reflexión como un proceso mediante el cual se da sentido a la práctica. Lo concibe como una forma de pensar sistemática, rigurosa y disciplinada que tiene sus raíces en la búsqueda científica (Simpson et al., 2004). Además, afirma que necesita darse en un contexto comunitario, es decir, en interacción con otros. Cuando nuestros docentes de inglés en formación se encuentran inmersos en los principios del aprendizaje experiencial, sus procesos están vinculados a una experiencia concreta interesante, son motivados a reflexionar sobre la misma, conceptualizar la manera de resolver una tarea y a experimentar soluciones. Ellos, a su vez, diseñan secuencias didácticas que lleven a sus estudiantes a una dinámica congruente con dichos principios. Para lograr lo anterior, se generan las distintas etapas del ciclo de Kolb (2009) explicadas anteriormente. A continuación, se describe cómo desde la asignatura de Didáctica del Idioma Inglés, se logra este continuo que inicia con experiencias concretas vinculadas a la realidad. Además, se crean espacios para que tanto estudiantes como docentes examinen y reflexionen sobre sus propios valores.

Con el fin de desarrollar habilidades de planificación e implementación de secuencias didácticas utilizando marcos teóricos congruentes con el aprendizaje experiencial, se realiza un proceso que va desde planeación y simulación de lo planificado en el aula de clases hasta la implementación de las mismas en el centro de práctica respectivo. El estudiantado tiene la posibilidad de presentar sus planificaciones mientras es observado por sus pares y la docente (experiencia concreta). Se llena una matriz (ver figura 2) que llamamos DAPPA, por las iniciales describir (observación reflexiva), analizar (conceptualización abstracta) y plan de acción (experimentación activa). En la primera columna, se registra lo que se observa; en la segunda, se analiza y teoriza sobre cómo funcionó la actividad y por qué, mientras que en la última se hace (en base a lo anterior) una sugerencia concreta de cómo mejorar los resultados de lo actuado. Como se señalaba, esto es realizado tanto por el estudiante mismo (en una autoevaluación reflexiva luego de implementar su plan de clase), como por los pares (a manera de coevaluación reflexionada) y por la docente (quien heteroevalúa fundamentando lo sugerido). En la figura 2, puede apreciarse cómo se realiza este proceso reflexivo.

Figura 2. Matriz DAPPA

\begin{tabular}{|l|l|l|}
\hline \multicolumn{1}{|c|}{ DESCRIBE } & \multicolumn{1}{|c|}{ ANALYZE } & PLANNED ACTION \\
\hline $\begin{array}{l}\text { 9:35 A. greets class and asks if they like } \\
\text { games. They say yes. She elicits some and } \\
\text { explains game (a balloon will be passed } \\
\text { and whoever has it when music stops, will } \\
\text { stand up and write a verb on the board. } \\
\text { "There are 9 students and all of them seem } \\
\text { engaged." }\end{array}$ & $\begin{array}{l}\text { Outstanding job at introducing } \\
\text { topic, } \\
\text { eliciting knowledge from them. } \\
\text { According to what we know } \\
\text { about learning, this allows you to } \\
\text { diagnose strengths and weaknesses } \\
\text { to be addressed during the rest of } \\
\text { the lesson. }\end{array}$ & Continue activating schema! \\
\hline
\end{tabular}




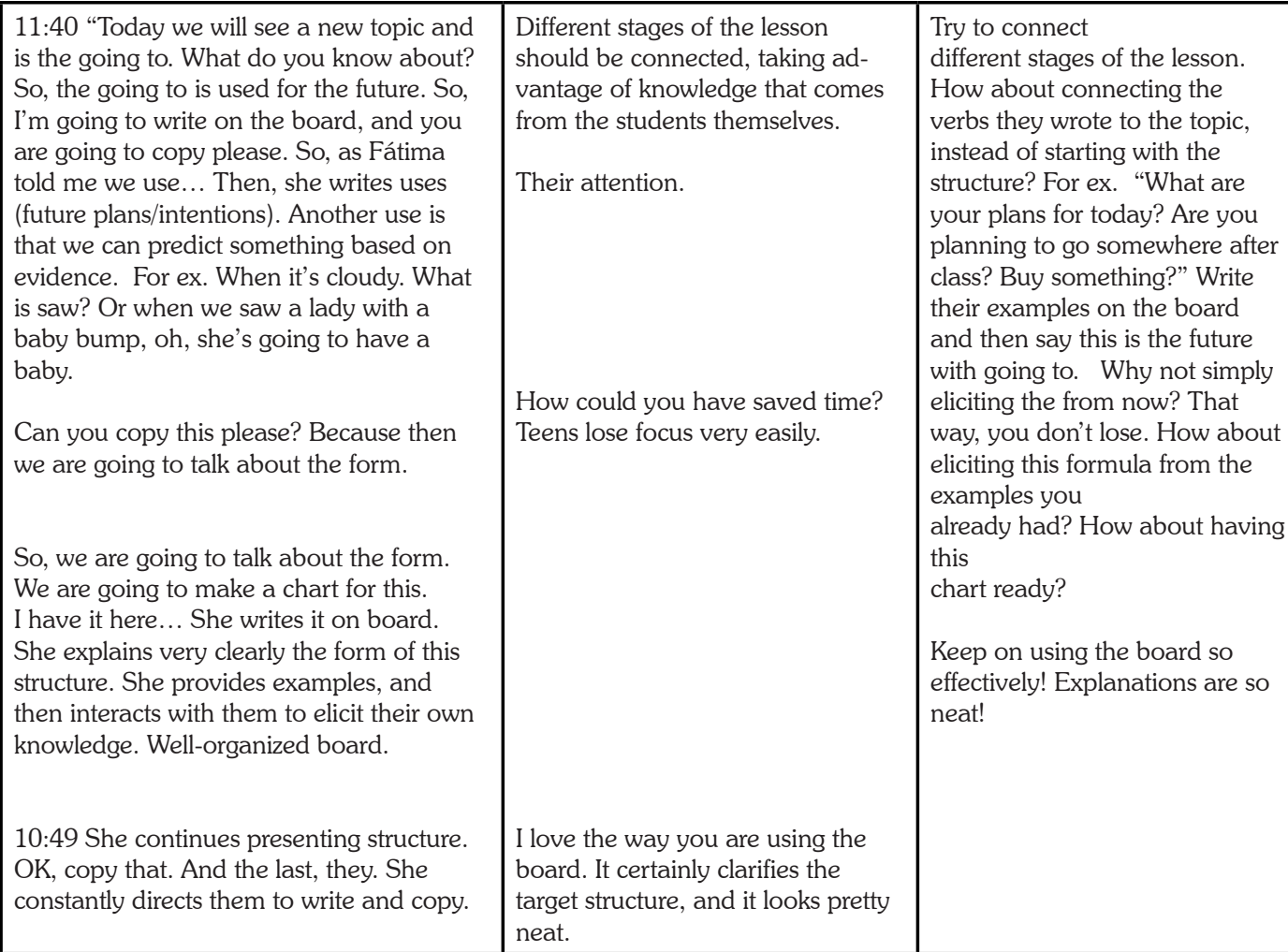

Funte: elaboración propia.

Como formadora de futuros formadores en idioma inglés, el carácter reflexivo del aprendizaje experiencial resulta muy práctico $e$ invita a concebir el aprendizaje como un proceso continuo que se ve potenciado por la reflexión, el análisis y la experimentación que le acompañan. Además, la utilización de un formato como el DAPPA hace que la práctica sea reflexionada con fundamentos teóricos y concretos que permiten regresar a esa realidad a implementar lo aprendido. El resultado es la introyección de una práctica docente reflexiva que parte de realidades concretas y regresa a ellas, con el fin de seguirlas transformando.

\section{Conclusiones}

Las experiencias desarrolladas han permitido constatar que el enfoque de aprendizaje experiencial ha contribuido a la formación de docentes del Profesorado y la Licenciatura en Idioma Inglés de la UCA, pues fortalece su capacidad de abordar y transformar sus contextos educativos de manera creativa, innovadora y crítica. Dentro de este enfoque, el estudiante es expuesto a experiencias de aprendizaje significativas y apoyadas por un ciclo de reflexión, análisis crítico y experimentación. Aun cuando el contenido de aprendizaje sigue siendo importante, el corazón del aprendizaje experiencial no es el producto, sino el proceso, además de la posibilidad de colaborar, aprender con y de los otros (Kolb, 2009).

A partir de las cuatro experiencias descritas, se concluye que esta metodología permite a profesionales de idioma inglés en formación resolver problemas de su realidad educativa y social de manera innovadora, 
especialmente en un contexto en el que la educación ha experimentado cambios vertiginosos y se requiere de respuestas a fenómenos permeados por la incertidumbre y la ambigüedad (Griffiths y Soruç, 2020). Esto se logra mediante el desarrollo de herramientas sociocognitivas, tales como el pensamiento crítico, el pensamiento creativo, las habilidades colaborativas y comunicativas.

El aprendizaje experiencial desarrolla capacidades didáctico-pedagógicas que hacen a nuestro estudiantado en formación más abierto a los procesos de aprendizaje y al cambio, lo cual genera posibilidades de transformación en las instituciones en que se desempeñan. Entre las habilidades más importantes se encuentran el diseño de secuencias didácticas y recursos de aprendizaje centrados en el estudiante, atendiendo sus diversas formas de aprender y distintas dimensiones de su ser (lo cognitivo, sí, pero también lo socioemocional). Esto favorece el desarrollo de competencias lingüísticas de sus estudiantes, rompiendo con la perspectiva de que la clase de inglés se da para monótonamente recitar frases descontextualizadas y llenar espacios en un libro de texto. En suma, los docentes y profesionales de inglés que han sido formados en aprendizaje experiencial desarrollan el potencial de incidir positivamente en el aprendizaje del idioma inglés de sus estudiantes, pues generan entornos educativos dinámicos $e$ innovadores que ponen al aprendiente y sus necesidades de aprendizaje al centro del proceso.

Lo experimentado-reflexionado-conceptualizado y reexperimentado reafirma la convicción que los principios del aprendizaje experiencial pueden cambiar radicalmente los contextos educativos en los que se implemente. En carreras vinculadas a la educación, se está frente a la posibilidad privilegiada de sembrar la semilla de una nueva cultura pedagógica en la que se conciba el aprendizaje del idioma inglés como una oportunidad para desarrollar habilidades interculturales, humanistas, éticas. Desde una perspectiva humanística y cristiana de la educación, lo más impor- tante es encontrar medios formativos para que esos conocimientos derivados de la ciencia y la tecnología sirvan como instrumentos de vida y esperanza de nuestros pueblos, y que nuestros egresados se apropien de ellos con esa mirada. Este enfoque educativo tiene mucho que aportar a la deconstrucción de paradigmas que despojan al lenguaje de su rol fundamental: crear y recrear la realidad.

\section{Referencias bibliográficas}

Ambrose, S., Bridges, M., DiPietro, M., Lovett, M. \& Norman, M. (2010). How learning works: 7 research-based principles for smart teaching. Jossey-Bass.

Badillo Torres, D. A. (2021). Adaptación y validación de un instrumento para medir flexibilidad cognitiva de estudiantes de $4 .^{\circ}$ a 6. año básico [Tesis de Magíster]. Pontificia Universidad Católica de Chile. https://repositorio.uc.cl/handle/11534/51220

Bartle, E. (2015). Experiential learning: an overview. Institute for Teaching and Learning Innovation. The University of Queensland.

Consorcio de Habilidades Indispensables para el Siglo XXI (2009). Logros indispensables para los estudiantes del siglo XXI [Web]. Eduteka. https://eduteka.icesi.edu.co/articulos/ SeisElementos

Cooper, L., Orrell, J. \& Bowden, M. (2010). Work Integrated Learning: A guide to effective practice. Routledge.

Fry, H., Ketteridge, S. \& Marshall, S. (2009). A handbook for teaching and learning in higher education: Enhancing academic practice (3rd. Ed.). Routledge.

Griffiths, C. \& Soruç, A. (2020). Language learning strategies. En Individual Differences in Language Learning (pp. 113-129). Palgrave Macmillan.

Harmer, J. (2001). The Practice of English Language Teaching (4th. Ed.). Pearson Longman. 
Haynes, C. (2007). Experiential learning: Learning by doing. http://adulteducation. wikibook.us/index.php?title= Experiential Learning_-_Learning_by_Doing

Kolb, D. (1984). Experiential learning: experience as the source of learning and development. Prentice Hall.

Kolb, D. (1976). The Learning Style Inventory. McBer and Company.

Kolb, D. \& Fry, R. (1975). Toward an applied theory of experiential learning. En C. Cooper (Ed.), Theories of Group Processes (pp. 33-57). John Wiley.

Kolb, A. \& Kolb, D. (2009). The Learning Way: Meta-cognitive Aspects of Experiential Learning. Simulation Gaming, 40(3), 297-327. https://journals.sagepub.com/ doi/10.1177/1046878108325713

Ministerio de Educación [MINED]. (2020). Desarrollo socioemocional: Orientaciones para la familia. https://www.mined.gob. sv/materiales/f3/semana8/socioemocional/ Orientaciones_familia_Sociomocional_I_ciclo_ f3.pdf

Pilbeam, R., Conley, Q., Christopherson, R. \& Barrus, A. (2012). BrainBook: Instructional Design Leveraging Social Networking Features Increases Motivation and Engagement. En T. Amiel \& B. Wilson (Eds.), Proceedings of EdMedia 2012 - World
Conference on Educational Media and Technology (pp. 1159-1166). Association for the Advancement of Computing in Education.

Riener, C. \& Willingham, D. (2010). The myth of learning styles. Change: The Magazine of Higher Learning, 42(5), 32-35. https://www.researchgate.net/publication/249039450_The_Myth_of_Learning_ Styles

Simpson, D. J., Jackson, M. J. \& Simpson, J. C. (2004). John Dewey and the art of teaching: Toward reflective and imaginative practice. Sage Publications.

Organización de las Naciones Unidas para la Educación, la Ciencia y la Cultura y Oficina Regional de Educación para América Latina y el Caribe [UNESCO y OREALC]. (2017). Reporte E2030: Educación y habilidades para el siglo XXI. Reunión Regional de Ministros de Educación de América Latina y el Caribe, Buenos Aires, Argentina, 24 y 25 de enero de 2017. https://bibliotecadigital.mineduc. $\mathrm{cl} /$ bitstream/handle/20.500.12365/17284/ E2030\%20EducacionyhabilidadesSigloXXI. pdf? sequence $=1 \&$ isAllowed $=y$

Wright, J. (2015). Experiential learning: An overview. Institute for Teaching and Learning Innovation. https:/itali.uq.edu.au/files/1264/ Discussion-paper-Experiential_learning_an_ overview.pdf 